\title{
The bicyclic form of galacto-noeurostegine is a potent inhibitor of $\beta$-galactocerebrosidase
}

Agnete H. Viuff, ${ }^{a}$ Stéphane Salamone, ${ }^{a}$ Joseph McLoughlin, ${ }^{\mathrm{b}}$ Janet E. Deane ${ }^{*}, \mathrm{~b}$ and Henrik H. Jensen ${ }^{*}, \mathrm{a}$

aDepartment of Chemistry, Aarhus University, Langelandsgade 140, 8000 Aarhus C, Denmark.

${ }^{b}$ Department of Clinical Neuroscience, Cambridge Institute of Medical Research, University of Cambridge, Hills Rd, Cambridge CB2 OXY, UK.

\section{Materials and Methods}

Galacto-noeurostegine (5, GNS) was prepared through chemical synthesis as previously described. ${ }^{1}$

\section{Enzyme inhibition}

Inhibition constants $\left(K_{\mathrm{i}}\right)$ were determined by measuring initial rates $(<10 \%$ substrate conversion $)$ using $p$-nitrophenyl $\beta$-D-galactopyranoside at six concentrations ranging from $1 / 4 K_{\mathrm{m}}$ up to $4 K_{\mathrm{m}}$. Reactions were conducted in $20 \mathrm{mM}$ sodium acetate, $150 \mathrm{mM} \mathrm{NaCl}, 0.1 \%$ vol/vol Nonidet P-40 (pH 4.6) at $25^{\circ} \mathrm{C}$. At eight time points $(15 \mathrm{sec}) 100 \mu \mathrm{L}$ aliquots of the reaction were transferred to a clear, flat bottomed, 96-well plate with $100 \mu \mathrm{L}$ stopping buffer $(\sim 350 \mathrm{mM} \mathrm{NaOH})$ and the resulting absorbance measured at $410 \mathrm{nM}$ using a PerkinElmer 2300 EnSpire Multilabel Plate Reader. $K_{\mathrm{m}}$ and $K_{\mathrm{m}}$ ' values were obtained by fitting to the equation $\mathrm{v}=$ $\left(\mathrm{V}_{\max }[\mathrm{S}]\right) /\left(K_{m}+[\mathrm{S}]\right)$ using the program GraphPad Prism 6. Competitive inhibition was established from Hanes plots. $K_{i}$ values were calculated as $K_{i}=[\mathrm{I}] /\left(\left(K_{\mathrm{m}}^{\prime} / K_{\mathrm{m}}\right)-1\right)$ having $[\mathrm{I}] \approx K_{\mathrm{i}}$. 
Michaelis-Menten plot

Galactocerebrosidase (mouse)

$g$ a lacto-Noeuros teg in e

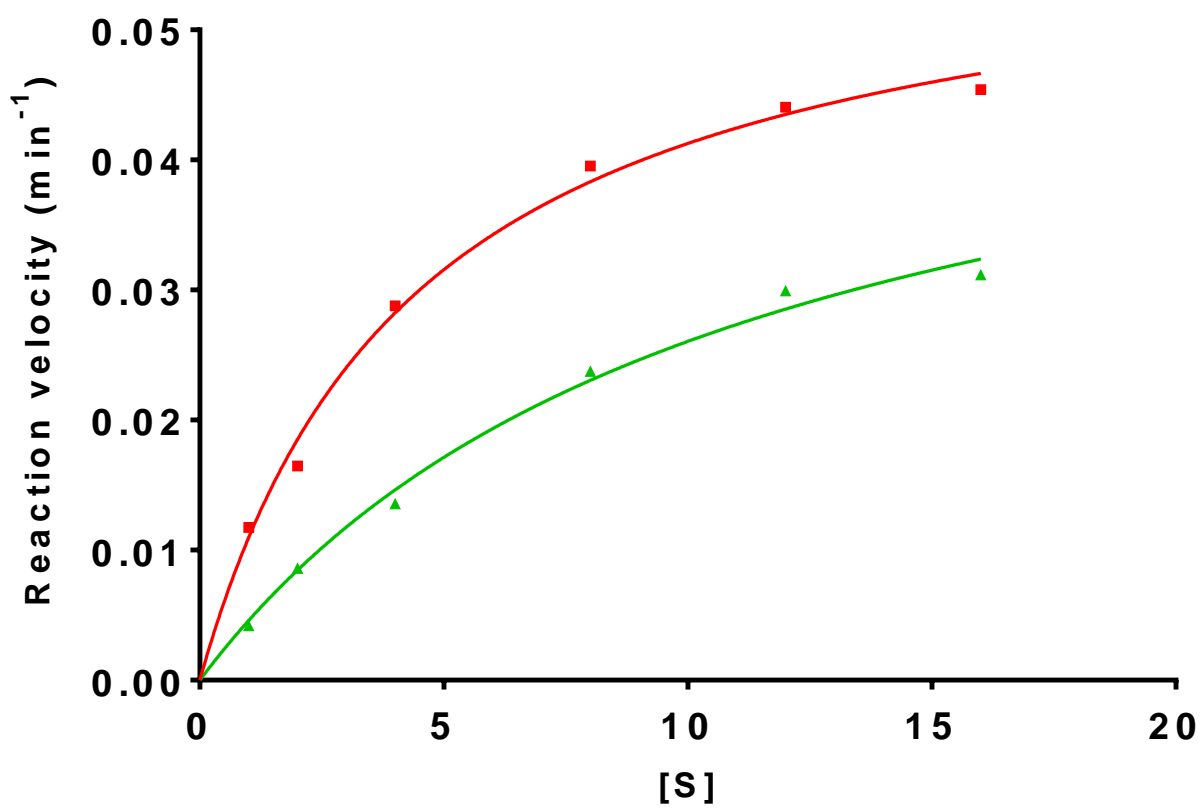

- No Inhibitor

$\neq 10$ u M

Hanes-W olf plot

Galactocerebrosidase (mouse)

galacto-Noeurostegine

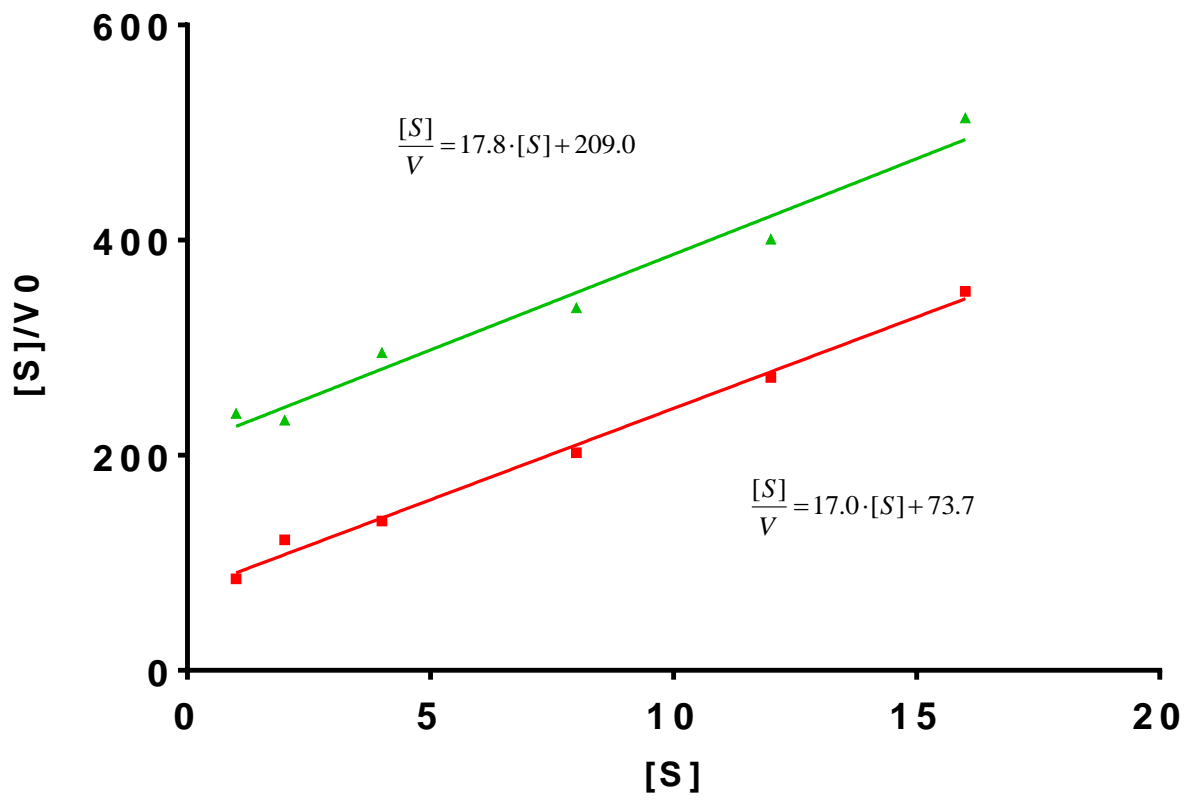

- No Inhibitor

$\neq 10$ uM 


\section{Protein expression, purification and crystallization}

GALC from mouse (mGALC) was expressed, purified and crystallized as described previously. ${ }^{2}$ Briefly, mGALC was expressed in HEK293T cells with an $\mathrm{N}$-terminal secretion signal and $\mathrm{His}_{6}$-tag. Protein was purified using $\mathrm{Ni}$ affinity chromatography. Purified protein was concentrated to $2.5 \mathrm{mg} / \mathrm{mL}$ in $150 \mathrm{mM} \mathrm{NaCl}$ and $10 \mathrm{mM}$ Hepes ( $\mathrm{pH}$ 7.4) and crystallized using sitting drop vapor diffusion against a reservoir of $0.2 \mathrm{M}$ sodium acetate, $0.1 \mathrm{M}$ sodium cacodylate ( $\mathrm{pH} 6.8$ ), and $34 \% \mathrm{wt} / \mathrm{vol}$ polyethylene glycol 8000 . Soaks were initiated by adding $0.5 \mu \mathrm{L}$ of 10 mM GNS ligand made up in reservoir solution (for $\mathrm{pH}$ 6.8) or reservoir solution supplemented with $230 \mathrm{mM}$ sodium acetate (for $\mathrm{pH} 4.6$ ), directly to the crystallization drop. Immediately after adding ligand solution, $0.5 \mu \mathrm{L}$ of perfluoropolyether oil was layered over the drop. Crystals were soaked at room temperature for 10-60 mins before being cryoprotected by removal from the drop through perfluoropolyether oil before flash-cooling in liquid nitrogen.

\section{Data collection, structure determination and refinement}

Diffraction data was recorded on the I04 beamline at Diamond Light Source at $100 \mathrm{~K}$ and $\lambda=0.98 \AA$ using a $6 \mathrm{M}-\mathrm{F}$ detector (Pilatus). Integration and scaling was performed using DIALS ${ }^{3}$ (Table 1) and the resolution cut-off was decided by $\mathrm{CC}_{1 / 2}$ value of approximately 0.5 and $\mathrm{I} / \sigma \mathrm{I}$ of $>1.0$ in the outer resolution shell. The reflections excluded from refinement ("FreeR") were identical to those used previously for the unliganded GALC structure. ${ }^{4}$ Unbiased electron-density maps of GALC-ligand complexes were generated following rigid-body refinement in phenix.refine ${ }^{5}$ using the native unliganded GALC as the input model (PDB ID 3ZR5). Protein Data Bank files and CIF restraints for GNS were generated from alphanumeric SMILES strings using eLBOW. ${ }^{6}$ All further refinement was performed iteratively using COOT $^{4}$ and phenix.refine. Structural figures were rendered using PyMOL (Schrödinger LLC). Hydrogen-bonding graphical representations were created using LIGPLOT+.?

\section{Differential scanning fluorimetry of mGALC}

Two $\mu \mathrm{g}$ of mGALC was prepared in $1 \mathrm{M}$ sodium acetate ( $\mathrm{pH} 4.6)$, MES ( $\mathrm{pH} 5.6$ and $\mathrm{pH} 6.6$ ) or PBS ( $\mathrm{pH} 7.4$ ) and mixed with $1 \times$ Thermal Shift Dye (Applied Biosystems) and either $0 \mathrm{mM}$ or $0.5 \mathrm{mM}$ GNS. Fluorescence was monitored using a Viia 7 Real-Time PCR System between $20^{\circ} \mathrm{C}$ and $95^{\circ} \mathrm{C}$ in $1{ }^{\circ} \mathrm{C}$ steps, with $20 \mathrm{~s}$ equilibration time per step. The melting temperature $\left(\mathrm{T}_{\mathrm{m}}\right)$ was the inflexion point of the sigmoidal curve obtained by curve fitting using GraphPad Prism version 5.0. Stabilization is expressed as the $\Delta \mathrm{T}_{\mathrm{m}}$ compared to a protein-only control. All experiments were performed in triplicate.

Table S1. Data Collection and Refinement Statistics. Values in parentheses are for highest-resolution shell.

\begin{tabular}{l|l|l}
\hline Data collection & GALC-GNS pH 6.8 & GALC-GNS pH 4.6 \\
\hline Wavelength $(\AA)$ & 0.9795 & 0.9795 \\
\hline Space group & R32 & R32 \\
\hline
\end{tabular}




\begin{tabular}{|c|c|c|}
\hline Cell dimensions & & \\
\hline$a, b, c(\AA)$ & $248.7,248.7,77.3$ & $250.8,250.8,77.9$ \\
\hline Resolution $(\AA)$ & $124.36-2.10(2.14-2.10)$ & $41.98-2.25(2.29-2.25)$ \\
\hline$R_{\text {merge }}$ & $0.153(1.790)$ & $0.154(2.518)$ \\
\hline$R_{\text {pim }}$ & $0.051(0.645)$ & $0.051(0.825)$ \\
\hline $\mathrm{CC}_{1 / 2}$ & $0.997(0.461)$ & $0.998(0.524)$ \\
\hline$I / \sigma I$ & $8.2(1.1)$ & $8.8(1.1)$ \\
\hline Completeness (\%) & $100(99.2)$ & $100(100)$ \\
\hline Multiplicity & $10.0(8.6)$ & $10.1(10.2)$ \\
\hline \multicolumn{3}{|l|}{ Refinement } \\
\hline Resolution $(\AA)$ & $56.07-2.10(2.14-2.10)$ & $42.0-2.25(2.30-2.25)$ \\
\hline No. reflections & $53021(2628)$ & $44225(2614)$ \\
\hline$R_{\text {work }} / R_{\text {free }}$ & $0.163 / 0.201$ & $0.159 / 0.198$ \\
\hline \multicolumn{3}{|l|}{ No. atoms } \\
\hline Protein & 5126 & 5100 \\
\hline Ligand & 13 & 13 \\
\hline Ions & 2 & 2 \\
\hline Water & 267 & 247 \\
\hline
\end{tabular}




\begin{tabular}{l|l|l}
\hline B-factors & & \\
\hline Protein & 52.1 & 57.8 \\
\hline Ligand & 35.5 & 42.1 \\
\hline Ions & 68.9 & 81.9 \\
\hline Water & 50.2 & 57.7 \\
\hline Ramachandran & & \\
\hline Favored $(\%)$ & 96.6 & 95.9 \\
\hline Outliers $(\%)$ & 0.3 & 0.8 \\
\hline r.m.s. deviations & & 0.007 \\
\hline Bond lengths $(\AA)$ & 0.07 & \\
\hline Bond angles $\left({ }^{\circ}\right)$ & 0.842 & \\
\hline PDB ID & $6 Y 6 S$ & \\
\hline
\end{tabular}

References 


\footnotetext{
${ }^{1}$ Salamone, S.; Clement, L. L.; Viuff, A. H.; Andersen, O. J.; Jensen, F.; Jensen, H. H. Synthesis and evaluation of galactonoeurostegine and its 2-deoxy analogue as glycosidase inhibitors. Org. Biomol. Chem. 2015, 13, 7979-7992.

${ }^{2}$ Hill, C. H.; Graham, S. C.; Read, R. J.; Deane, J. E. Structural snapshots illustrate the catalytic cycle of $\beta$-galactocerebrosidase, the defective enzyme in Krabbe disease. Proc. Natl. Acad. Sci. U. S. A. 2013, 110, 20479-20484.

${ }^{3}$ Winter, G.; Waterman, D. G.; Parkhurst, J. M.; Brewster, A. S.; Gildea, R. J.; Gerstel, M.; Fuentes-Montero, L.; Vollmar, M.; Michels-Clark, T.; Young, I. D.; Sauter, N. K.; Evans, G. DIALS: implementation and evaluation of a new integration package. Acta Crystallogr. D: Biol. Crystallogr. 2018, 74, 85-97.

${ }^{4}$ Deane, J. E.; Graham, S. C. Kim, N. N.; Stein, P. E.; McNair, R.; Cachón-Gonzáles, M. B.; Cox, T. M.; Read, R. J. Insights into Krabbe disease from structures of galactocerebrosidase. Proc. Natl. Acad. Sci. U. S. A. 2011, 108, 15169-15173.

${ }^{5}$ Afonine, P. V.; Grosse-Kunstleve, R. W.; Echols, N.; Headd, J. J.; Moriaty, N. W.; Mustyakimov, M.; Terwilliger, T. C.;

Urzhumtsev, A.; Zwart, P. H.; Adams, P. D. Towards automated crystallographic structure refinement with phenix.refine. Acta Crystallogr., D: Biol. Crystallogr. 2012, 68, 352-367.

${ }^{6}$ Moriary, N. W.; Grosse-Kunstleve, R. V.; Adams, P. D. electronic Ligand Builder and Optimization Workbench (eLBOW): a tool for ligand coordinate and restraint generation. Acta Crystallogr., D: Biol. Crystallogr. 2009, 65, 1074-1080.

${ }^{7}$ Laskowski, R. A.; Swindells, M. B. LigPlot+: Multiple Ligand-Protein Interaction Diagrams for Drug Design. J. Chem. Inf. Model. 2011, 51, 2778-2786.
} 